Cita: Zanetti, M.C.; Feltran, G.N.; Dias, H.M.; Polito, L.F.T.; Souza Júnior, L.A.; Villas Boas

Junior, M.; Filgueiras, I.P.; Brandão, M.R.F. (2019). Efeito de um programa de apoio às necessidades psicológicas básicas sobre os comportamentos pró e anti-socias na educação física escolar. Cuadernos de Psicología del Deporte, Vol 19(2), 70-82

\title{
Efeito de um programa de apoio às necessidades psicológicas básicas sobre os comportamentos pró e anti-socias na educação física escolar
}

\author{
Efecto de un programa de apoyo a las necesidades psicológicas básicas \\ sobre los comportamientos pró y anti-sociales en la educación física \\ escolar
}

\section{Effect of a program to support basic psychological needs on pro and} antisocial behavior in school physical education

\author{
Zanetti, M.C. ${ }^{1,2}$; Feltran, G.N. ${ }^{1}$; Dias, H.M. ${ }^{1}$; Polito, L.F.T. ${ }^{1}$; Souza Júnior, L.A. ${ }^{1,2}$; Villas Boas Junior, \\ M. ${ }^{1}$; Filgueiras, I.P. ${ }^{1}$; Brandão, M.R.F. ${ }^{1}$ \\ ${ }^{1}$ Universidade São Judas; ${ }^{2}$ Universidade Paulista
}

\begin{abstract}
RESUMO
O objetivo foi verificar o efeito de uma intervenção, fundamentada no atendimento das necessidades psicológicas básicas (NPB) e comportamentos pró e antissociais em aulas de Educação Física escolar. Participaram deste estudo 45 alunos e 2 professores de educação Física divididos em Grupo Controle (GC) e Intervenção (GI). Os alunos responderam o Questionário de Atitudes no Esporte (QAE-23) e participaram de uma entrevista semiestruturada nos momentos pré e pós-intervenção. A intervenção foi realizada com o professor do GI durante 10 semanas, com objetivo de promoção das NPB durante as aulas. Foi utilizado o teste T de Student e adotado $p<0,05$. As entrevistas foram categorizadas e analisadas. Observou-se percepção do atendimento das NPB e redução de comportamentos antissociais. Conclui-se que a intervenção pautada no cumprimento das NPB possibilitou redução dos comportamentos antissociais, porém, a duração do estudo pode ter sido um fator limitante de maior efeito em relação à internalização desses comportamentos.

Palabras clave: Teoria da Autodeterminação; Necessidades Psicológicas Básicas; Comportamentos antissociais; Educação física escolar
\end{abstract}

\section{RESUMEN}

El objetivo fue verificar el efecto de una intervención, fundamentada en la atención de las necesidades psicológicas básicas (NPB) y comportamientos pro y antisociales en clases de Educación Física escolar. Participaron de este estudio 45 alumnos y 2 profesores de educación física divididos en Grupo Control (GC) e Intervención (GI). Los alumnos respondieron el Cuestionario de Actitudes en el Deporte (QAE-23) y participaron en una entrevista 


\section{Zanetti, M.C., Feltran, G.N., Dias, H.M., Polito, L.F.T., Souza Júnior, L.A., Villas Boas Junior, M., Filgueiras, I.P., Brandão, M.R.F.}

semiestructurada en los momentos pre y post-intervención. La intervención fue realizada con el profesor del GI durante 10 semanas, con objetivo de promoción de las NPB durante las clases. Se utilizó la prueba T de Student y se adoptó $\mathrm{p}<0,05$. Las entrevistas fueron categorizadas y analizadas. Se observó percepción de la atención de las NPB y reducción de comportamientos antisociales. Se concluye que la intervención pautada en el cumplimiento de las NPB posibilitó reducción de los comportamientos antisociales, sin embargo, la duración del estudio puede haber sido un factor limitante de mayor efecto en relación a la internalización de esos comportamientos.

Palabras clave: Teoría de la autodeterminación; Necesidades Psicológicas Básicas; Comportamientos antisociales; Educación física escolar.

\section{ABSTRACT}

The objective was to verify the effect of an intervention, based on the attendance of the basic psychological needs (NPB) and pro and antisocial behaviors in school physical education classes. Thirty-five students and two teachers of Physical Education, divided into Control Group (CG) and Intervention (GI) participated in this study. The students answered the Attitudes Questionnaire in Sport (QAE-23) and participated in a semi-structured interview in the pre- and post-intervention moments. The intervention was performed with the GI teacher for 10 weeks, aiming to promote NPB during class. The Student's t-test was used and adopted $p<0.05$. The interviews were categorized and analyzed. It was observed perception of NPB care and reduction of antisocial behaviors. It was concluded that intervention based on NPB compliance enabled a reduction of antisocial behavior, but the duration of the study may have been a limiting factor of greater effect in relation to the internalization of these behaviors.

Keywords Self-Determination Theory; Basic Psychological Needs; Antisocial behaviors; School physical education

\section{INTRODUÇÃO}

A autodeterminação é o conjunto de comportamentos, esforços e ações intencionais que possibilitam ao indivíduo ser o agente causal de suas escolhas e que o mobilizam a interagir com o ambiente ou realizar uma determinada tarefa, de modo que sejam satisfeitas suas necessidades (Appel, Wendt, \& Argimon, 2010; E. Deci \& Ryan, 1985; Wehmeyer, Shogren, Little, \& Lopez, 2017). Nesse sentido, a teoria da Autodeterminação (TAD), proposta por Deci e Ryan (1985), busca compreender as causas e consequências dos comportamentos autodeterminados, isto é, como as pessoas agem e se comprometem com suas atividades por vontade própria.

Para tanto, a TAD destaca três Necessidades Psicológicas Básicas (NPB): autonomia, competência e relação social. A autonomia está atrelada à ação de autogovernar-se, ou seja, são as escolhas e decisões realizadas em conformidade com os valores pessoais e com adequado nível de reflexão e consciência. Por sua vez, a competência implica na necessidade de ser eficaz em suas interações com o ambiente, ou a percepção do indivíduo de se sentir capaz de realizar algo. Por fim, a relação social é a construção de relações afetivas, procura por relacionamentos com outras pessoas, grupos ou comunidades, em busca da atividade de amar e ser amado. Destarte, a satisfação das NPB pode levar a comportamentos autodeterminados e percepção de bem-estar (Appel et al., 2010; E. Deci \& Ryan, 1985; Moreno; Martínez, 2006). No entanto, o desenvolvimento da autonomia pode resultar em satisfação das outras duas NPB, pois um contexto que ofereça suporte à autonomia poderá ser propício à aprendizagem e ao desempenho, com efeitos diretos na motivação, considerados importantes para a internalização de valores e regras, que propiciarão às pessoas maior autodeterminação (Balaguer, Castillo, \& Duda, 2008; Deci \& Ryan, 2008).

Observa-se que a TAD investiga fatores que facilitam a motivação, integração social e bem-estar e, alternativamente, aqueles que contribuem para comportamentos antissociais (Ryan \& Deci, 2017). O comportamento antissocial pode ser definido como ações que infringem ou transgridam regras sociais, violação de direitos, condutas agressivas, delinquência, distúrbios de conduta e de personalidade (Carvalho \& Gomide, 2005; Gequelin \& Carvalho, 2007). Nesse sentido, Patterson, DeBaryshe, and Ramsey (1989) sugerem em seu 


\section{Programa de apoio às NPB e prosocialidade na educação física escolar}

modelo teórico que o comportamento antissocial está associado a desajustes na interação familiar e aspectos sociais que influenciam o processo de desenvolvimento da criança. Os autores apontam que a negligência e más práticas parentais podem originar comportamentos desordenados, que culminam no fracasso escolar, assim como a rejeição pelos pares no contexto social. Em última análise, pressupõe-se que as crianças inseridas nesse contexto correm alto risco de evasão escolar e/ou de casa, assim como o envolvimento em situações crônicas de delinquência.

Atualmente, os comportamentos antissociais no ambiente escolar, os quais se manifestam de maneira intensa e por meio de agressões físicas, verbais e simbólicas (Abramovay \& Rua, 2002), tornaram-se eventos recorrentes e têm alarmado professores, diretores, coordenadores, pais e até os próprios alunos (Carvalho \& Gomide, 2005). Nesse sentido, durante as aulas de Educação Física escolar (EFE), não raro, ocorrem situações agressivas envolvendo os alunos, que se revelam por meio de cobranças exageradas entre colegas e que, por muitas vezes, estão fora dos limites do bom senso por não alcançar os resultados desejados (Kaminski \& El Tassa, 2010).

Nesse sentido, a literatura (Mountjoy et al., 2016; Ribeiro \& de Azevedo, 2018; Torres \& de Araújo, 2015) reporta diversas situações que envolvem o comportamento agressivo, tanto no contexto da EFE, como no contexto esportivo. Em relação a esse fato, Machado (2016) argumenta que o comportamento agressivo e violento dos indivíduos envolvidos está diretamente ligado à importância e envolvimento emocional que a atividade representa para cada indivíduo. Todavia, o ambiente das aulas de EFE deveria ser um espaço diferenciado, no qual, através de suas propostas e estratégias pedagógicas, representasse um espaço de possibilidades concretas de interação e de aprendizagem, em que o conviver e o divertir-se com o outro pudessem ser valorizados, ajudando na assimilação das regras sociais de convivência (Almeida \& Freire, 2014; Baliulevicius \& Macário, 2006; Campillo, Zafra, \& Redondo, 2008).

Destarte, cabe ao professor, enquanto mediador durante as aulas de EFE, minimizar e reduzir comportamentos antissociais, assim como propiciar um ambiente que desenvolva comportamentos autodeterminados e ações positivas tais como o respeito pelos seus pares, autocontrole e cooperação, que pode também ser fruto do atendimento das NPB (Armour, Sandford, \& Duncombe, 2013; Reeve, 2002). Nesse sentido, estratégias adotadas pelo professor durante as aulas de Educação Física escolar pautadas no atendimento das necessidades psicológicas básicas podem influenciar de forma positiva ou negativa o comportamento do aluno e contribuir para sua motivação (Arantes da Costa, Borsato Passos, Matias de Souza, \& Fiorese Vieira, 2017; Carraça, 2017; Taylor, Ntoumanis, \& Standage, 2008). Visto que o atendimento das NPB está relacionado a um ambiente promissor durante as aulas, qual seria o efeito de um programa de intervenção nos comportamentos pró e antissociais em aulas de EFE? Portanto, o objetivo da presente investigação é verificar o efeito de um programa de intervenção com vistas ao fomento das NPB dos alunos, nos comportamentos pró e antissociais em aulas de EFE.

\section{MATERIAL E MÉTODOS}

A presente pesquisa caracteriza-se como estudo descritivo que apresenta as características do objeto de estudo a partir de dados primários (GIL, 2008). De acordo com Samara e Barros (2002; p. 30), "os dados da pesquisa descritiva, podem ser obtidos originalmente, a partir de entrevistas pessoais ou de discussão em grupo, relacionando e confirmando as hipóteses levantadas na definição do problema da pesquisa". Já Gil (2007), complementa que a pesquisa descritiva tem como objetivo principal a descrição das características de determinada população ou de determinado fenômeno, ou o estabelecimento de relação entre as variáveis.

Uma vez que o objetivo da pesquisa científica é descrever, interpretar, explicar ou predizer a realidade, a utilização de métodos quantitativos e qualitativos apresentam suas potencialidades e limitações, contribuindo de forma específica com a pesquisa (Paranhos, Figueiredo Filho, Rocha, Silva Júnior, \& Freitas, 2016). No entanto, em função da necessidade de reunir dados estatísticos com informações textuais, a literatura (Johnson \& Onwuegbuzie, 2004; Mayoh \& Onwuegbuzie, 2015; Onwuegbuzie, Gerber, \& Schamroth Abrams, 2017) denota a tendência em utilizar de forma integrada as abordagens, técnicas, métodos e concepções 


\section{Zanetti, M.C., Feltran, G.N., Dias, H.M., Polito, L.F.T., Souza Júnior, L.A., Villas Boas Junior, M., Filgueiras, I.P., Brandão, M.R.F.}

quantitativas e qualitativas, definida como métodos mistos, as quais podem possibilitar um desenho de pesquisa mais robusto, assim como favorecer uma melhor forma de compreender os fenômenos pesquisados e combinar a abordagem indutiva com a lógica dedutiva (Camerino, Castañer, \& Anguera, 2014; Creswell, 2010; Paranhos et al., 2016).

Devido à complexidade do fenômeno e a interação das variáveis envolvidas optou-se pela utilização de métodos mistos, por meio da abordagem concorrente (Camerino et al., 2014), na qual os dados foram coletados de forma simultânea..

\section{O estabelecimento de ensino e participantes}

A pesquisa foi realizada em uma escola pública da rede estadual de ensino da cidade de São José do Rio Pardo- SP, que atende alunos do $6^{\circ}$ ao $9^{\circ}$ ano do ensino fundamental, médio e ensino supletivo. A escola possui aproximadamente 1021 alunos, sendo 398 alunos matriculados no ensino fundamental, 518 no ensino médio e 105 no ensino supletivo. O quadro docente é composto por 56 professores de diversas disciplinas, sendo três deles professores de Educação Física, 14 funcionários, uma vice-diretora e um diretor.

A escolha da instituição de ensino se justifica pelo fato de conter as amostras convenientes para a pesquisa, ou seja, ter dois professores de EFE diferentes no mesmo ano, porém, ministrando aula em diferentes turmas, a fim de evitar a contaminação das amostras, uma vez que, inconscientemente o professor de Educação Física poderia usar as informações do Grupo Intervenção (GI) no Grupo Controle (GC), causando uma possível interferência nos resultados obtidos ao final da pesquisa. Após a escolha da instituição participante, foi agendada uma reunião com o diretor responsável pela instituição, com a intenção de informar ao mesmo, os objetivos da pesquisa e obter sua autorização para o estudo.

Em relação aos professores de EFE, o professor do GI tem 39 anos e se formou no ano de 2007, efetivo na rede estadual e docente há oito anos. Atualmente, reside e trabalha no mesmo munícipio em que leciona, com jornada de trabalho de 2 horas/aulas, no período vespertino. $\mathrm{O}$ segundo participante é o professor do GC de 46 anos, formado no ano de
1995, efetivo na rede estadual e docente há 21 anos. Também reside e trabalha no mesmo município em que leciona, com jornada de trabalho de 26 horas/aulas, distribuídas entre duas escolas públicas.

Foram sujeitos da pesquisa 45 alunos, divididos em 2 diferentes grupos por meio de sorteio: Grupo Controle (GC) e Grupo Intervenção (GI). Dessa forma, o GC foi composto por 18 alunos, sendo 13 do sexo masculino, com idade média de $13,38 \pm 0,49$ e 05 do sexo feminino com idade média de $13,40 \pm 0,49$. Por sua vez, o GI foi composto por 24 alunos, sendo 15 alunos do sexo masculino com idade média de $13,40 \pm 0,49$ e 09 do sexo feminino com idade média de $13,56 \pm 0,50$.

\section{Instrumentos}

Para verificar a efetividade do programa no atendimento das NPB dos alunos foi realizada uma entrevista semiestruturada. Essa escolha se justifica pelo fato de que esse tipo de instrumento consiste em um meio de elencar de forma mais abrangente possível as questões na qual o pesquisador quer abordar, a partir de suas hipóteses provenientes da definição do objeto de investigação (MINAYO, 1999). A entrevista semiestruturada foi realizada de forma individual em uma sala de aula, com duração aproximada de 40 minutos. Foram realizadas duas entrevistas: 1) antes da intervenção e 2) após a intervenção. O roteiro da entrevista realizada antes da intervenção consistiu em questões relacionada às rotinas e atividades das aulas de educação física, em relação à promoção de autonomia, competência e relação social, assim como a ocorrência de comportamentos antissociais dos colegas e a consequente intervenção do professor. A entrevista realizada após 10 semanas consistiu em questões relacionadas às mudanças e comparações dessas mesmas situações e experiências ao decorrer da intervenção. Destarte, a percepção dos alunos em relação às atividades com ênfase nas NPB e situações de comportamentos antissociais durante as aulas de EFE foram categorizadas em: 1) Autonomia; 2) Competência; 3) Relação social e 4) Atitudes pró e antissociais . Todas as entrevistas foram gravadas por meio de um gravador de voz digital da marca Sony ${ }^{\circledR}$, modelo ICD-PX240-4gb. 


\section{Programa de apoio às NPB e prosocialidade na educação física escolar}

Também foi utilizado o Questionário de Atitudes no Esporte (QAE-23) que avalia a concordância com as atitudes declaradas nos itens do questionário. $\mathrm{O}$ instrumento é composto por 23 itens, distribuídos de forma aleatória e relacionados a quatro dimensões atitudinais no esporte. Há duas dimensões consideradas pró-sociais (empenho e convenção) e duas dimensões consideradas antissociais (trapaça e antidesportivísmo). A dimensão empenho se faz presente nas afirmativas de $\mathrm{n}^{\mathrm{o}} 01,06,11,15,21$; a dimensão convenção nas afirmativas de $\mathrm{n}^{\circ} 04,08,12$, 17, 22; a dimensão trapaça nas afirmativas de $\mathrm{n}^{\circ} 03$, $05, \quad 09, \quad 10,13,16,19,23 ; \quad$ a dimensão antidesportivismo nas afirmativas de $\mathrm{n}^{\circ} 02,07,14$, 18,20 . As respostas ao questionário foram dadas por meio de uma escala tipo likert, com graduação de cinco pontos, indo de (1) "discordo firmemente da declaração" a (5) "concordo firmemente com a declaração". As propriedades métricas desse instrumento foram avaliadas no estudo de Gonçalves, Silva, Chatzisarantis, Lee, e Cruz (2006) para o contexto português.

\section{Procedimentos}

$\mathrm{O}$ projeto de pesquisa atendeu aos parâmetros éticos e recomendações da Resolução 466/12, além de ter sido aprovado pelo Comitê de Ética e Pesquisa da Universidade São Judas Tadeu - SP, no dia 20 de abril de 2016 sob o número: CEP. $\mathrm{N}^{\circ}$ 53827216.0.0000.0089.

No primeiro contato com os alunos foram explicitados os objetivos da pesquisa e entregue aos alunos em duas vias o Termo de Consentimento Livre e Esclarecido (TCLE), que devia ser assinado pelos pais. Para aqueles que apresentaram a autorização foi explicitado sobre o preenchimento do QAE-23 e também foi realizada uma entrevista semiestruturada de forma individual com cerca de $10 \%$ dos alunos sorteados em ambos os grupos (GC e GI). Após a aplicação inicial dos instrumentos e da entrevista foi realizada uma intervenção de orientação semanal durante 10 semanas, com o objetivo de realizar adaptações na aula planejada a fim de levar os alunos ao suporte das NPB. Essas sessões de orientação aconteciam semanalmente, junto com a pesquisadora e antes das aulas de EFE. Adicionalmente, essas reuniões eram realizadas somente com o professor de EFE do GI, enquanto que no GC não foi realizada nenhuma intervenção pela pesquisadora. As discussões estabelecidas durante as reuniões semanais tiveram como propósito elaborar estratégias, atividades e exercícios, pautados no planejamento atual do professor e que pudessem colaborar para que suas aulas fossem fundamentadas no cumprimento das NPB.

Com o intuito de promover maior autonomia aos alunos, foi oportunizado que eles realizassem escolhas e tomassem decisões durante as atividades propostas no planejamento do professor, as quais eram apresentadas e discutidas por todos. Dessa forma, dava-se oportunidade de discutir quem seria o capitão para escolher times ou até mesmo, durante a partida de o grupo decidir a melhor estratégia para solucionar possíveis desafios impostos pela atividades, oferecer oportunidades para todos pudessem apontar seu ponto de vista e opinar em eventuais mudanças de regras. Para tanto, o professor precisou adaptar atividades, atendendo às sugestões dos alunos, bem como as suas preocupações e necessidades. Dessa forma, foram expostos a importância dessas atividades e os seus benefícios; utilizando um diálogo não controlador e dando chance ao aluno de expressar suas ideias. Buscando promover a competência dos alunos, as tarefas foram adequadas ao nível de desenvolvimento dos alunos, pois se as atividades fossem muito complexas os alunos poderiam sentir dificuldades e consequentemente incompetentes. As atividades propostas deveriam ser desafiantes, trazendo um desejo ao participante de superar seus próprios obstáculos, aumentando gradualmente seus conhecimentos. Adicionalmente, durante os momentos finais da aula foi proposta uma auto avaliação aos alunos, indagando aos mesmos como avaliavam seus desempenhos. $O$ intuito desta estratégia era permitir ao aluno perceber sua competência, possibilitando feedbacks positivos para avançar em suas aprendizagens. Por fim, também foram incentivadas atividades que promovessem maior relação social entre os alunos, a fim de contribuir para com o processo de pertencimento e apoio social durante as aulas. Ao final das dez semanas foi realizada a entrevista e aplicação do QAE-23 com os mesmos alunos, de ambos os grupos, que participaram no início da pesquisa.

No primeiro encontro com o professor foi discutido a organização das aulas e ficou estabelecido que o 


\section{Zanetti, M.C., Feltran, G.N., Dias, H.M., Polito, L.F.T., Souza Júnior, L.A., Villas Boas Junior, M., Filgueiras, I.P., Brandão, M.R.F.}

conteúdo nas duas primeiras aulas fosse o futsal. Assim, com o intuito de favorecer autonomia, a turma escolheu uma atividade de aquecimento no início das aulas, as equipes, os capitães dos times e distribuiu das funções de mesários e juízes. Dessa forma, todos foram envolvidos no jogo, buscando com que os mesmos se sentissem competentes. No segundo encontro foram discutidas pesquisas sobre o papel do professor como motivador dos alunos e ficou estabelecido que durante a próxima aula os alunos pudessem realizar uma lista de atividades de acordo com o planejamento do professor.

No terceiro encontro, a aula possuiu conteúdo teórico-prático e a temática a ser trabalhada foi o atletismo, com atividades práticas adequadas ao nível de desenvolvimento do aluno. Durante a aula teórica, o professor realizou esclarecimentos das atividades e permitiu que os alunos colaborassem com as explicações desenvolvidas.

No quarto encontro, a aula teve objetivo de interação com toda a comunidade escolar para a importância da adoção de um estilo de vida mais ativo, saudável e feliz, mediante o aumento da prática de atividade física diária. Nesse dia foi desenvolvida uma gincana com outras salas de aula com intuito de promover a cooperação e maior relação social entre os alunos.

No quinto encontro foi discutido o envolvimento dos alunos nas aulas e a importância da motivação do professor $\mathrm{e}$ de fomentar junto aos alunos $\mathrm{o}$ cumprimento das NPB durante as aulas. A temática da aula foi os aspectos táticos do futsal com a proposta de trabalhar atividades mais próximas daquelas já conhecidas pelos alunos e consequentemente que promovessem maior percepção de competência. Também foi realizado jogos populares com envolvimento de todo o grupo, estabelecendo a relação social.

No sexto, sétimo e oitavo encontro a temática proposta foi o basquete com atividades que promovessem maior interação entre os alunos, além de evitar a exposição de habilidades mais complexas e que pudessem levar à diminuição da percepção de competência. Foi fomentada a autonomia com participação dos alunos na escolha das atividades, divisão das equipes e distribuição das funções.
No nono encontro, foi oportunizada aos alunos a organização da aula livre, com o objetivo de promover autonomia, na qual todos pudessem realizar prática prazerosa com a máxima participação dos alunos.

\section{Análisis estadístico}

As entrevistas semiestruturadas foram gravadas e em seguida transcritas de forma literal, com seus respectivos códigos, seguindo as recomendações de Bardin (2011), que organiza a análise de conteúdo em três fases fundamentais: 1) pré análise; 2) exploração do material ; 3) tratamento dos resultados, inferência e interpretação.

A pré análise consistiu na leitura geral a partir da transcrição das entrevistas e organização do material nas quatro etapas: 1) leitura flutuante (na qual o pesquisador tomou conhecimento do texto e transcreveu as entrevistas); 2) escolha dos documentos (seleção do que seria analisado); 3) formulação de hipóteses e objetivos (formulação de afirmações provisórias, que o pesquisador se propôs a verificar); 4) elaboração de indicadores (recortes de textos no documento analisado, em relação aos temas que mais se repetiam).

A segunda fase compreendeu na Exploração do material, que consistiu na codificação e na definição das categorias, na identificação das unidades de registros e das unidades de contextos dos documentos. Nessa fase, foram realizados recortes dos textos em unidades de registros (correspondeu ao segmento de conteúdo, temas, palavras ou frases), realizando a primeira categorização. Em seguida, as categorias foram agrupadas de acordo com os temas, que originaram as categorias intermediárias, as quais foram classificadas e agrupadas em função das temáticas que resultaram nas categorias finais.

Já a terceira e última fase, consistiu nas intepretações das categorias, ou seja, no tratamento dos resultados, inferência e interpretação. Nesta etapa ocorreu a condensação e o destaque das informações para análise, culminando nas interpretações inferenciais. Esta fase representou o momento da intuição da análise reflexiva e crítica, a qual possibilitou interpretar as categorias embasando-se nos conceitos 


\section{Programa de apoio às NPB e prosocialidade na educação física escolar}

estudados pelos autores (Bardin, 2011). No entanto, esse procedimento mereceu alguns cuidados, pois as categorias devem ser: homogêneas, exaustivas (esgotar a totalidade do texto), exclusivas (um mesmo elemento do conteúdo não pode ser classificado em duas categorias), objetivas e adequadas ou pertinentes. Dessa forma, nesta pesquisa foi utilizado o critério de categorização semântica, ou seja, categorização temática (Bardin, 2011).

Os dados quantitativos, provenientes do QAE-23, foram armazenados e tratados no pacote estatístico SPSS versão 20.0. A fim de identificar possíveis diferenças entre grupos (GI e GC) já existentes antes e após a intervenção, foi aplicado um teste $\mathrm{T}$ para amostras independentes. Para verificar se houve diferença no GI e GC entre os momentos pré e após a intervenção foi aplicado um teste $\mathrm{T}$ para amostras dependentes. O nível de significância adotado foi de $5 \%$. Foi explorado também o percentual de variação (delta relativo) do pós-teste para o pré-teste entre as variáveis mensuradas.

\section{RESULTADOS}

Em relação aos dados qualitativos, relacionado ao atendimento das NPB e obtidos pelas entrevistas, a formação das categorias foi realizada após a triagem do material e a leitura flutuante, sendo selecionados os trechos das falas dos entrevistados. Nesta pesquisa foi utilizado o critério de categorização semântico, ou seja, categorização temática (Bardin, 2011), que resultou em 4 (quatro) diferentes categorias: Autonomia, Competência, Relação social e Atitudes pró e antissociais na EFE. Com intuito de melhor descrever a análise dos dados, tais categorias foram pautadas nas narrativas dos entrevistados pré e póscoleta, nas quais os alunos entrevistados foram identificados pela letra "S", por ordem numeral $(1,2$, 3 e 4) e em relação ao grupo (Grupo Intervenção; GC: Grupo Controle).

Ao verificar a categoria "Autonomia" os relatos denotam maior liberdade oferecida pelo professor ao GI Pós (S1, S2, S3, S4), para opinar, discutir e organizar propostas de atividades relevantes às aulas, conforme relato do S3 GI Pós: "Sim, por que antes de descer pra quadra ele perguntava o que a gente quer jogar e a gente resolvia tudo dentro da classe para ficar tudo pronto". Ao analisar o GC, percebe-se que a autonomia do (S1, S2 e S3) foi proporcionada por meio do sistema de "aula livre" (quando o professor permite ao aluno escolher suas atividades sem nenhuma intervenção), de acordo com o relato do S3 GC Pós: "Sim de um mês pra cá, depois que ele deu tudo o que queria, ele deixa a gente fazer aula livre". "A gente escolhe o que quer e ele autoriza a gente fazer". Porém, nota-se que S4 GC Pós, relata "Às vezes ele pergunta o que a gente quer fazer, daí eu acho bom porque a gente aprende mais e também mais gente participa", demonstrando possuir maiores níveis de motivação quando o professor oferece liberdade na escolha das atividades.

Em relação à NPB competência verificou-se que os sujeitos do GI Pós (S1, S2, S3 e S4) sentiram-se competentes e seguros ao realizar as tarefas propostas pelo PGI. Além de competência, as atividades parecem ter deixado os alunos mais motivados para realizarem os exercícios conforme relata S3 GI Pós: “... me liberei mais e antes eu só gostava do basquete, aí comecei fazer o futsal e aprendi a gostar também pelos exercícios que o professor ensinava, porque mudou a aula e se eu fizesse só o jogo acho que eu ia ficar mais parada". Também é possível notar que os sujeitos do GC (S2, S4) sentiram-se competentes pela justificativa de gostar de fazer a aula de EFE. Nesse sentido, (S1) relata que sua competência se dá quando ele pode auxiliar a aprendizagem de seus colegas e (S3) sente-se competente pois seu PGC propõe atividades de coordenação.

$\mathrm{Na}$ categoria "Relacionamento Social" observou-se que alunos do GI Pós (S2, S3 e S4) notaram mudança na organização do trabalho do professor, a fim de buscar atividades significantes e prazerosas. Cabe evidenciar o relato de S4 GI Pós: “... tipo assim, se um não quer jogar ninguém joga, porque não dá. Daí o professor conversa com a gente pra arrumar isso, né!” Já S1 GI Pós, relatou haver maior envolvimento entre todos devido ser a atividade que todos gostam de fazer, o futebol. Em relação aos alunos do GC (S2, $\mathrm{S} 3$, S4), os mesmos relataram que houve envolvimento de todos, pois sempre jogam juntos e misturados entre ambos os sexos. Evidencia-se o relato de S4 GC Pós: “... no futebol e no vôlei todos participam”. Já S1 GC Pós relata que as meninas permanecem sentadas e os meninos estão sempre unidos jogando futebol, não havendo maior envolvimento dos mesmos durante a aula. 


\section{Zanetti, M.C., Feltran, G.N., Dias, H.M., Polito, L.F.T., Souza Júnior, L.A., Villas Boas Junior, M., Filgueiras, I.P., Brandão, M.R.F.}

A respeito dos dados sobre os comportamentos antissociais, foram obtidos dados quantitativos e qualitativos. Os dados quantitativos relativos às dimensões pró-sociais (empenho e convenção) e antissociais (trapaça e antidesportivismo), obtidos Tabela 1

Comparação dos escores das dimensões pró-sociais e antissociais entre os grupos e momentos pré e pós intervenção.

\begin{tabular}{lcccccc}
\hline & \multicolumn{2}{c}{ Grupo Controle $(\mathbf{n = 1 8})$} & \multicolumn{2}{c}{ Grupo Intervenção (n=24) } \\
\cline { 2 - 7 } & Pré-teste & Pós-teste & $\boldsymbol{p}$ & Pré-teste & Pós-teste & $\boldsymbol{p}$ \\
\hline Empenho & $19,16 \pm 5,33$ & $19,50 \pm 6,41$ & 0,816 & $20,83 \pm 5,48$ & $18,58 \pm 9,04$ & 0,309 \\
Convenção & $18,16 \pm 5,67$ & $16,33 \pm 5,95$ & 0,339 & $18,12 \pm 7,40$ & $16,29 \pm 8,36$ & 0,401 \\
Trapaça & $17,17 \pm 6,43$ & $12,72 \pm 6,24$ & 0,056 & $19,50 \pm 8,73$ & $15,87 \pm 10,80$ & 0,203 \\
Antidesportivismo & $10,89 \pm 5,83$ & $8,89 \pm 4,12$ & 0,271 & $13,04 \pm 6,35$ & $9,54 \pm 6,73$ & 0,058 \\
\hline
\end{tabular}

pelas respostas do QAE-23 são apresentados na tabela 1.
Em relação ao resultado das dimensões avaliadas pelo QAE verificou-se que não houve diferença estatisticamente significativa entre os grupos (GI e GC) e momentos de avaliação (Pré e Pós) em relação aos níveis de atitudes pró e antissociais nas aulas de Educação Física escolar. Este resultado pode estar relacionado à duração do estudo (10 semanas), o qual pode ter sido um fator limitante de maior efeito em relação à ausência de alteração verificada por meio QAE-23. Nesse sentido, Korpershoek, Harms, de Boer, van Kuijk e Doolaard (2016) sugerem pesquisas com maior prazo de intervenção, pois programas de intervenções mais extensivos podem possibilitar verificar os efeitos de manutenção dessas estratégias no comportamento e no desenvolvimento sócio emocional dos alunos.

Ao analisar os dados qualitativos relacionados à categoria "Atitudes Pró e Antissociais na EFE" percebeu-se que alunos do GI Pós (S1, S2 e S4) não notaram nenhuma discussão ou agressão física durante as aulas de EFE. Para S3 GI Pós aconteceram discussões em outros momentos e com outra classe: "Só quando as meninas foram jogar com a outra classe, que não deu muito certo, porque a outra classe tomava muito nossa bola e ainda machucava muito a gente e daí a gente investia nelas também". Ao analisar relatos de alunos do GC Pós (S1, S2, S3) foram citadas discussões quando surgia algum tipo de desentendimento durante as competições. Cabe evidenciar o relato de S1 GC Pós: "Quando a gente compete sempre tem uma briguinha e discussões 


\section{Programa de apoio às NPB e prosocialidade na educação física escolar}

pós-intervenção, como forma de verificar a manutenção desses efeitos e comportamentos mesmo após cessado o programa.

\section{DISCUSSÃO}

Observa-se que tão importante quanto o conteúdo das aulas são as estratégias metodológicas adotadas, que promovam e contribuam para o desenvolvimento de um cidadão mais autônomo, pois, ao permitir maior autonomia aos alunos, os professores promovem possibilidades de escolher, discutir, refletir e organizar-se na prática. Assim, a promoção da autonomia nos ambientes escolares contribui e reforça os comportamentos intrinsecamente motivados, ou seja, um sujeito autônomo, motivado intrinsecamente se torna o regulador de seus comportamentos sem necessidade de influências causadas por fatores externos (Standage, Duda, \& Ntoumanis, 2005; Zanetti et al., 2017).

Moy, Renshaw e Davids (2016) sugerem que os alunos podem apresentar sentimentos positivos, persistirem nas atividades em que realizam e encontrarem satisfação em sua aprendizagem quando percebem que possuem autonomia, o domínio da atividade (competência) e que são apoiados por colegas e professores. Destarte, Deci e Ryan (2000) destacam que os sujeitos que regulam autonomamente a motivação, demonstram maior persistência, empenho, esforço e prazer nas práticas ou atividades nas quais realiza. Portanto, contextos que privilegiam a autonomia por meio de estratégias organizadas pelo professor de EFE propiciam oportunidades aos alunos de escolher, criar e adaptar regras, levando a indivíduos mais responsáveis e participativos nas tarefas que realizam.

Observa-se que as crianças que se percebem altamente competentes em um determinado domíniocognitivo, sócio afetivo, e/ou motor, podem demonstrar prazer no processo de aprendizagem, persistência frente às dificuldades, possibilidades de resolver os problemas de forma independente e ainda demonstram sentimentos de confiança em suas tarefas (Valentini, 2006). Ntoumanis e Standage (2009) apontam que os alunos também se percebem competentes quando seu professor não estabelece a comparação com os demais alunos do grupo e nesse sentido, tal situação pode ser observada no relato do S3 GI Pós, quando comenta que sua motivação aumentou a partir do momento que se sentiu competente na tarefa que experimentou. Assim, podemos constatar que as crianças que apresentaram maior competência nas atividades, sentiram-se autoconfiantes em suas habilidades, aceitando desafios em suas práticas, estimulando a motivação e consequentemente a permanência nas atividades.

Nesse sentido, as estratégias de intervenção do professor de EFE são essenciais para promover mudanças comportamentais, pois ao adotar uma metodologia que favoreça a competência, os alunos tendem a melhorar seus níveis de habilidades e seu desempenho e consequentemente pode levar ao desenvolvimento de comportamentos autodeterminados (Koka \& Hagger, 2010). À medida que suas metodologias/estratégias derem suporte às NPB (autonomia, competência e relação social), maiores possibilidades de aumentar a motivação e consequências positivas como maior nível de saúde física e mental (Silva, Barata, \& Teixeira, 2013; Torregrosa, Belando, \& Moreno-Murcia, 2014).

Observa-se que a interação entre professor e aluno apresenta papel fundamental no processo de aprendizagem, pois além de construírem um ambiente mais prazeroso, pode motivar e oportunizar o desenvolvimento do aluno (Reeve, Jang, Carrell, Jeon, \& Barch, 2004). Fatores sociais como estilo interpessoal ou estilo de ensino dos professores podem contribuir para uma possível motivação dos sujeitos, pois atribuem aos alunos oportunidades para que se sintam relacionados quando interagem dentro de um ambiente social que oferece afeição e atenção (Deci \& Ryan, 2000; Skinner \& Edge, 2002).

Para tanto, Tollefson (2000) sugere que a desmotivação durante as aulas pode ser atribuída a diversos fatores como a competição entre os alunos, aulas desinteressantes promovidas pelo professor, inabilidade do professor para ensinar e motivar, a revolta em situações de derrota em um jogo competitivo ou rebeldia e agressividade frente a situações do cotidiano das aulas. Neste sentido, a forma que o professor dialoga com seus alunos e apresenta seus conteúdos influencia a maneira que estes irão vivenciar a atividade, pois se o professor promover uma linguagem não controladora $\mathrm{e}$ informativa aos alunos, haverá maiores chances dos mesmos de terem feedbacks positivos no desenvolvimento das aulas. 


\section{Zanetti, M.C., Feltran, G.N., Dias, H.M., Polito, L.F.T., Souza Júnior, L.A., Villas Boas Junior, M., Filgueiras, I.P., Brandão, M.R.F.}

Adicionalmente, ao analisar os discursos, observouse no relato de alunos de ambos os grupos que as agressões físicas eram raras e as agressões verbais eram mais recorrentes. A esse respeito, García-Calvo, Sánchez-Oliva, Leo, Amado e Pulido (2016) consideram que a violência verbal é a mais utilizada entre os alunos, independente do sexo e em alguns casos, os termos utilizados não são considerados ofensas, mas sim, vícios de linguagem próprios da faixa etária. Todavia, para que tudo ocorra dentro do planejamento esperado pelo professor é necessário que se deixem claras as regras e os limites das atividades propostas, além estabelecer que estes devam ser respeitados.

Esta gestão da aula pode ser definida como as ações que os professores realizam para criar um ambiente de apoio à aprendizagem e favorecer aos alunos a auto regulação de seus comportamentos (Marzano, Marzano, \& Pickering, 2003). Nesse sentido, observa-se que o professor é responsável por motivar seus alunos, propiciando um ambiente saudável e agradável, capaz de superar qualquer conflito. Destarte, para minimizar os comportamentos antissociais, pode-se recorrer ao gerenciamento de sistemas de regras e rotinas dos alunos no início do ano letivo, relacionado à organização dos materiais, respeito, tolerância, empatia e ajuda aos colegas de classe (García-Calvo et al., 2016).

Em recente metanálise, Korpershoek et al. (2016) observaram que intervenções relacionadas à regulação do comportamento e desenvolvimento sócio emocional apresentam efeitos positivos em alunos no ensino fundamental. De fato, a satisfação das NPB é precursora de motivação intrínseca para a prática esportiva, que por sua vez, pode favorecer o comportamento positivo e um ambiente de respeito com os colegas e professores. Por outo lado, a frustração dessas NPB pode ocasionar motivação mais controlada ou exibição de atitudes antissociais. Destarte, o suporte à autonomia fornecido pelo professor está positivamente relacionado aos comportamentos pró sociais e negativamente relacionado aos comportamentos antissociais (Hodge \& Lonsdale, 2011; Ntoumanis \& Standage, 2009; Ryan \& Deci, 2017).

\section{CONCLUSÃO}

Conclui-se, por meio dos relatos obtidos na entrevista, que a intervenção pautada no cumprimento das NPB possibilitou um efeito positivo em relação à redução dos comportamentos antissociais, porém, os dados quantitativos sugerem que a duração do estudo pode ter sido um fator limitante de maior efeito em relação à internalização desses comportamentos.

\section{APLICAÇÕES PRÁTICAS}

Verificou-se efeito positivo da intervenção realizada para fomentar as NPB dos alunos como forma de aumentar os comportamentos pró-sociais e diminuir os comportamentos antissociais durante as aulas de EFE. Tal resultado aponta para a relevância desse tipo de intervenção na formação de um ambiente mais saudável, acolhedor e até de desenvolvimento das potencialidades, por privilegiar a autonomia e competência dos alunos. Por outro lado, sugere-se intervenções mais longas como possibilidade de internalização desse comportamento.

\section{REFERÊNCIAS}

1. Abramovay, M., \& Rua, M. d. G. (2002). Violências nas escolas. Brasília: UNESCO.

2. Almeida, Z., \& Freire, E. S. (2014). Jogos cooperativos nas aulas de educação física: o envolvimento dos alunos. Revista Mackenzie de Educação Física e Esporte, 13(1).

3. Appel, M., Wendt, G. W., \& Argimon, I. I. d. L. (2010). A Teoria da Autodeterminação e as influências sócio-culturais sobre a identidade. Psicologia em Revista, 16(2), 351-369. https://doi.org/10.5752/P.16789563.2010v16n2p351

4. Arantes da Costa, L. C., Borsato Passos, P. C., Matias de Souza, V. d. F., \& Fiorese Vieira, L. (2017). Educação física e esportes: motivando para a prática cotidiana escolar. Movimento, 23(3). 


\section{Programa de apoio às NPB e prosocialidade na educação física escolar}

5. Armour, K., Sandford, R., \& Duncombe, R. (2013). Positive Youth Development and Physical Activity/Sport Interventions: Mechanisms Leading to Sustained Impact. Physical Education and Sport Pedagogy, 18, 256-281. doi:10.1080/17408989.2012.666791

6. Balaguer, I., Castillo, I., \& Duda, J. L. (2008). Apoyo a la autonomía, satisfacción de las necesidades, motivación y bienestar en deportistas de competición: un análisis de la teoría de la autodeterminación. Revista de psicología del deporte, 17(1).

7. Baliulevicius, N. L. P., \& Macário, N. M. (2006). Jogos cooperativos e valores humanos: perspectiva de transformação pelo lúdico. Fitness $\&$ performance journal(1), 50-56. doi:10.3900/fpj.5.1.50.e

8. Bardin, L. (2011). Análise de conteúdo. Lisboa: Edições, 70.

9. Camerino, O., Castañer, M., \& Anguera, T. M. (2014). Mixed Methods Research in the Movement Sciences: Case studies in sport, physical education and dance (Vol. 5): Routledge.

10. Campillo, N. C., Zafra, A. O., \& Redondo, A. B. (2008). Relaciones entre la práctica de actividad física y el autoconcepto, la ansiedad y la depresión en chicas adolescentes. Cuadernos de psicología del deporte, 8(1), 61-77.

11. Carbelo, B. (2006). Estudio del sentido del humor: validación de un instrumento para medir el sentido del humor, análisis del cuestionario y su relación con el estrés. (Tesis doctoral). Universidad de Alcalá, Madrid.

12. Carraça, E. V. (2017). Um modelo motivacional do envolvimento dos jovens nas aulas de educação física. Retos: nuevas tendencias en educación física, deporte y recreación(31), 282291.

13. Carvalho, M. C. N., \& Gomide, P. I. C. (2005). Práticas educativas parentais em famílias de adolescentes em conflito com a lei. Estudos de psicologia, 22(3), 263-275.

14. Coll, C. (2010). Desarrollo, aprendizaje y enseñanza en la Educación Secundaria. Barcelona: Graó.
15. Creswell, J. W. (2010). Projeto de pesquisa métodos qualitativo, quantitativo e misto. Porto Alegre, RS: Artmed.

16. Deci, E. L., \& Ryan, R. M. (2000). The" what" and" why" of goal pursuits: Human needs and the self-determination of behavior. Psychological inquiry, 11(4), 227-268.

17. Deci, E. L., \& Ryan, R. M. (2008). Selfdetermination theory: A macrotheory of human motivation, development, and health. Canadian psychology/Psychologie canadienne, 49(3), 182.

18. Deci, E., \& Ryan, R. M. (1985). Intrinsic motivation and self-determination in human behavior: Springer Science \& Business Media.

19. Duckur, L. C. B. (2003). Em busca da formação de indivíduos autônomos nas aulas de educação física.

20. García-Calvo, T., Sánchez-Oliva, D., Leo, F. M., Amado, D., \& Pulido, J. J. (2016). Effects of an intervention programme with teachers on the development of positive behaviours in Spanish physical education classes. Physical Education and Sport Pedagogy, 21(6), 572-588. https://doi.org/10.1080/17408989.2015.1043256

21. Gequelin, J. L., \& Carvalho, M. C. N. (2007). Escola e comportamento anti-social. Ciências \& Cognição, 11.

22. Gonçalves, C. E., Silva, M. J. C., Chatzisarantis, N., Lee, M. J., \& Cruz, J. (2006). Tradução e validação do SAQ (Sports Attitudes Questionnaire) para jovens praticantes desportivos portugueses com idades entre os $13 \mathrm{e}$ os 16 anos. Revista Portuguesa de Ciências do Desporto, 6(1), 38-49.

23. González, J., Caprara, G. V., Garcés de los Fayos, E. J. y Zuffianó, A. (2014). Importance of the development of prosocial behavior in the adolescent's personality through physical education. En Physical Education: Role of School. Programs, Children's Attitudes and Health Implications. NOVA SCIENCE PUBLISHERS. New York.

24. Hodge, K., \& Lonsdale, C. (2011). Prosocial and antisocial behavior in sport: The role of coaching style, autonomous vs. controlled motivation, and moral disengagement. Journal of sport and 


\section{Zanetti, M.C., Feltran, G.N., Dias, H.M., Polito, L.F.T., Souza Júnior, L.A., Villas Boas Junior, M., Filgueiras, I.P., Brandão, M.R.F.}

exercise psychology, 33(4), 527-547. https://doi.org/10.1123/jsep.33.4.527

25. Johnson, R. B., \& Onwuegbuzie, A. J. (2004). Mixed methods research: A research paradigm whose time has come. Educational researcher, 33(7), 14-26. https://doi.org/10.3102/0013189X033007014

26. Kaminski, M., \& El Tassa, K. (2010). A prática pedagógica de educação física e a violência no contexto escolar. Curitiba: Secretaria da Educação Paraná Retrieved from http://www.diaadiaeducacao.pr.gov.br/portals/pd e/arquivos/2514-6.pdf.

27. Koka, A., \& Hagger, M. S. (2010). Perceived teaching behaviors and self-determined motivation in physical education: A test of selfdetermination theory. Research Quarterly for Exercise and Sport, 81(1), 74-86. https://doi.org/10.1080/02701367.2010.1059963 0

28. Korpershoek, H., Harms, T., de Boer, H., van Kuijk, M., \& Doolaard, S. (2016). A MetaAnalysis of the Effects of Classroom Management Strategies and Classroom Management Programs on Students' Academic, Behavioral, Emotional, and Motivational Outcomes. Review of Educational Research, 86(3), 643-680. doi:10.3102/0034654315626799

29. Machado, N. J. (2016). Educação: cidadania, projetos e valores. Sâo Paulo: Editora Escrituras.

30. Marzano, R. J., Marzano, J. S., \& Pickering, D. (2003). Classroom management that works: Research-based strategies for every teacher. Alexandria, VA: Association for Supervision and Curriculum Development.

31. Mayoh, J., \& Onwuegbuzie, A. J. (2015). Toward a conceptualization of mixed methods phenomenological research. Journal of mixed methods research, 9(1), 91-107.

32. MINAYO, M. C. S. (1999). Desafio do Conhecimento. Pesquisa Qualitativa em Saúde. São Paulo: Hucitec-Abrasco.

33. Moreno, J. A., \& Martínez, A. (2006). Importancia de la Teoría de la Autodeterminación en la práctica físico- deportiva: Fundamentos e implicaciones prácticas. Cuadernos de psicología del deporte, 6(2).

34. Mountjoy, M., Brackenridge, C., Arrington, M., Blauwet, C., Carska-Sheppard, A., Fasting, K., . . Budgett, R. (2016). International Olympic Committee consensus statement: harassment and abuse (non-accidental violence) in sport. British Journal of Sports Medicine, 50(17), 1019-1029. doi:10.1136/bjsports-2016-096121

35. Moy, B., Renshaw, I., \& Davids, K. (2016). The impact of nonlinear pedagogy on physical education teacher education students' intrinsic motivation. Physical Education and Sport Pedagogy, 21(5), 517-538. https://doi.org/10.1080/17408989.2015.1072506

36. Ntoumanis, N., \& Standage, M. (2009). Morality in sport: A self-determination theory perspective. Journal of Applied Sport Psychology, 21(4), 365380.

https://doi.org/10.1080/10413200903036040

37. Onwuegbuzie, A. J., Gerber, H. R., \& Schamroth Abrams, S. (2017). Mixed methods research. The International Encyclopedia of Communication Research Methods, 1-33.

38. Paranhos, R., Figueiredo Filho, B., Rocha Carvalho, E., da Silva Júnior, J. A., \& Freitas, D. (2016). Uma introdução aos métodos mistos. Sociologias, 18(42).

39. Patterson, G. R., DeBaryshe, B. D., \& Ramsey, E. (1989). A developmental perspective on antisocial behavior. Am Psychol, 44(2), 329-335.

40. Reeve, J. (2002). Self-determination theory applied to educational settings. In E. L. Deci \& R. M. Ryan (Eds.), Handbook of selfdetermination research (pp. 183-203). Rochester, NY: University Of Rochester Press.

41. Reeve, J., Jang, H., Carrell, D., Jeon, S., \& Barch, J. (2004). Enhancing students' engagement by increasing teachers' autonomy support. Motivation and emotion, 28(2), 147-169.

42. Ribeiro, E. P., \& de Azevedo, M. A. O. (2018). Empatia e Vontade de Vencer: Dois Polos em Tensão Permanente no Esporte. FairPlay, Revista 


\section{Programa de apoio às NPB e prosocialidade na educação física escolar}

de Filosofia, Ética y Derecho del Deporte(11), 68-95.

43. Ryan, R. M., \& Deci, E. L. (2017). Selfdetermination theory: Basic psychological needs in motivation, development, and wellness: Guilford Publications.

44. Silva, M. N., Barata, J. L. T., \& Teixeira, P. J. (2013). Exercício físico na diabetes: missão impossível ou uma questão de motivação? Revista portuguesa de cardiologia, 32, 35-43. https://doi.org/10.1016/S0870-2551(13)70046-5

45. Skinner, E., \& Edge, K. (2002). SelfDetermination, Coping, and Development. E. Deci, \& R. Ryan (1st Eds.), Handbook of SelfDetermination Research, 297-338.

46. Standage, M., Duda, J. L., \& Ntoumanis, N. (2005). A test of self- determination theory in school physical education. British Journal of Educational Psychology, 75(3), 411-433.

47. Taylor, I. M., Ntoumanis, N., \& Standage, M. (2008). A self-determination theory approach to understanding the antecedents of teachers' motivational strategies in physical education. Journal of sport and exercise psychology, 30(1), 75-94.

48. Tollefson, N. (2000). Classroom applications of cognitive theories of motivation. Educational Psychology Review, 12(1), 63-83.

49. Torregrosa, D., Belando, N., \& Moreno-Murcia, J. A. (2014). Predicción de la satisfacción con la vida en practicantes de ejercicio físico saludable. Cuadernos de psicología del deporte, 14(1), 117122.

50. Torres, N. V., \& de Araújo, E. L. (2015). A indisciplina e a violência: a redução a partir da prática da educação física na escola. ANAIS DO SCIENCULT, 6(1), 181-190.

51. Valentini, N. C. (2006). Competência e autonomia: desafios para a Educação Física Escolar. Rev. bras. Educ. Fís. Esp., São Paulo, 20, 185-187.

52. Wehmeyer, M. L., Shogren, K. A., Little, T. D., \& Lopez, S. J. (2017). Development of SelfDetermination Through the Life-Course. Dordrecht, Netherlands: Springer.
53. Zanetti, M. C., Feltran, G. N., Polito, L. F. T., Dias, H. M., Neves, A. N., \& Brandão, M. R. F. (2017). An intervention program on motivation and psychological needs in physical education. Revista de psicología del deporte, 26(4), 003438.Bay-Hinitz, A.K., Peterson, R.F. y Quilitch, H.R. (1994). Cooperative games: A way to modify aggressive and cooperative behaviors in young children. Journal of Applied Behavior Analysis, 27(3), 435-446. 